\title{
Frequency Domain Preamble-Based Channel Estimation and Equalization in LoRa
}

This paper was downloaded from TechRxiv (https://www.techrxiv.org).

\section{LICENSE}

CC BY 4.0

SUBMISSION DATE / POSTED DATE

$07-10-2021 / 12-10-2021$

\section{CITATION}

Savaux, Vincent; Savelli, Patrick (2021): Frequency Domain Preamble-Based Channel Estimation and Equalization in LoRa. TechRxiv. Preprint. https://doi.org/10.36227/techrxiv.16760101.v1

$\mathrm{DOI}$

10.36227/techrxiv.16760101.v1 


\title{
Frequency Domain Preamble-Based Channel Estimation and Equalization in LoRa
}

\author{
Vincent Savaux and Patrick Savelli \\ Advanced Connectivity Lab \\ $\mathrm{b}<>$ com, Rennes, France \\ Email: \{vincent.savaux,patrick.savelli\}@b-com.com
}

\begin{abstract}
This paper deals with multipath channel estimation and equalization in LoRa. It is suggested to take advantage of the cyclic property of the symbols in the LoRa frame preamble to obtain an interference-free version of the symbols in the frequency domain. Then, estimation methods used in multicarrier systems can be applied, such as the least square (LS), and the minimum mean square error (MMSE) estimators. It is shown that the cyclic property in LoRa is inherently independent of the length of the channel, making these estimation techniques robust to any frequency-selective channel. In addition the frequency domain zero-forcing (ZF) equalizer is used, and an original phase equalizer is introduced, taking advantage of the constant modulus property of LoRa symbols in the frequency domain. The performance of the investigated estimators and equalizers is shown through simulations, and applications to the presented results are further discussed.
\end{abstract}

Index Terms-LoRa, Channel Estimation, Equalization, Least Square, Minimum mean square error, Zero forcing.

\section{INTRODUCTION}

The low power wide area (LPWA) technologies of Internet of things (IoT) enable the massive connectivity of a constantly growing number of various devices and offer new applications, taking part in the digital transformation of industry verticals [1]-[3]. Among these LPWA technologies [4], [5], LoRa (for long range), developped by Semtech, is a widely used solution for the deployment of networks using non-licensed bands [6]. The LoRa signal is based on the so-called chirp spread spectrum modulation scheme, whose time and frequency specificity has been investigated in [7].

Due to the narrowband nature of the LoRa signal, the channel modelling is often omitted when designing signal processing algorithms, like the synchronization scheme for instance [8]-[10]. Otherwise, the channel is usually considered as a single coefficient assumed to be constant over all the bandwidth. This allows the authors of [11]-[14] to express the analytic error rate probability of the LoRa signal over different channel models, and to analyze the robustness of LoRa over varying channels [15]. In [16], the authors actually consider multipath channels in the application of LoRa frame detection. More generally, to the best of our knowledge, few papers such as [17] deal with multipath channel estimation in LoRa. However, it is likely that the propagation environment inherent to LPWAs is prone to induce non-negligible delayed echoes, if the device is situated far from the gateway or in deep underground for instance. These echoes should then be estimated and cancelled to recover proper LoRa symbols.
In this paper, we therefore tackle the issue of multipath channel estimation and equalization in LoRa. To this end, we express the discrete Fourier transform (DFT) of any modulated LoRa chirp, and we show that it is also a modulated chirp in the frequency domain. Then, we suggest to consider the cyclic property of the symbols in the preamble of the LoRa frame to rewrite the problem in the frequency domain, where the intersymbol interference vanishes. This result is similar to that in cyclic prefix-orthogonal frequency division multiplexing (CP-OFDM) modulation scheme. As a consequence, we can apply to LoRa some frequency channel estimation methods originally developed for CP-OFDM [18]-[21]. In this work, we focus on the least square (LS) and minimum mean square error (MMSE) estimators. Moreover, we take advantage of the $K$ symbols of the preamble to improve the LS estimator without any additional complexity. We show that, unlike CPOFDM, the channel estimation based on the LoRa preamble is robust against extremely long delay spread channel, as it is not limited by the $\mathrm{CP}$ length.

In addition, since any modulated LoRa symbol can be expressed in the frequency domain through the DFT, we suggest to perform the equalization in the frequency domain. Two methods are proposed: the simple one tap zero forcing (ZF) equalizer, and an original phase equalizer, based on the fact that a chirp in the frequency domain has a constant modulus and only its phase can then be equalized. More generally, since the modulated LoRa symbols are similar in both time and frequency domains, we suggest to perform the whole LoRa demodulation in the frequency domain, including channel estimation and equalization, and symbol detection. It is noteworthy that this is an original result and an interesting low complex alternative to the usual time domain demodulation [8], [11] (where the multipath channel is not considered) or the demodulation based on Fresnel transform (which is complex) [22], [23]. Finally, simulations results validate the suggested methods, and a discussion paves the way for further work.

The rest of the paper is organized as follows: Section II introduces the system model in time and frequency domains. Then, Section III shows how to take advantage of the cyclic property of the preamble to derive frequency domain channel estimators, and introduces the equalization methods as well. The performance of the presented estimation and equalization methods are investigated in Section IV, and a discussion is proposed in Section V, providing leads for future works. 
Finally, Section VI concludes this paper.

Notations: the normal font $a$ is used for scalar and the bold face $\mathbf{a}$ indicates matrices (including vectors). The parameters in time domain and frequency domain are highlighted by lower case a and capital letters $\mathbf{A}$, respectively. Moreover, $(.)^{-1}$, $(.)^{T}$, and $(.)^{H}$ refer to the inverse, transpose, and conjugate transpose (Hermitian) matrix operators, respectively.

\section{SySTEM MODEL}

The LoRa frame is composed of three main parts: the preamble, mainly used for the time and frequency synchronization (and frequency channel estimation in this paper), the header that carries control information (payload size, channel coding rate, etc.), and the payload that carries data. The modulation process of the data symbols in LoRa can be summarized as follows: the binary stream from the medium access control (MAC) layer is split into packets of size $S F$, where $S F$ is called the spreading factor (SF). The SF is a key parameter as it allows to adapt the LoRa symbol length, and therefore its robustness against distortions, to the cost of a data rate loss. Then, the binary packet is mapped onto a symbol $s^{(m)}, m \in\left\{0,1, . ., 2^{S F}-1\right\}$, which can be expressed as

$$
s^{(m)}(t)=\exp \left(j \pi \frac{B_{w}}{T_{s}}\left(t+\left(\frac{2 m}{N}-1\right) T_{s}\right) \cdot t\right),
$$

where $t \in\left[0, T_{s}\right], T_{s}$ is the symbol duration, $B_{w}$ is the bandwidth of LoRa signal, typically $125 \mathrm{kHz}$ (but $250 \mathrm{kHz}$ and $500 \mathrm{kHz}$ are available as well), such that $B_{w} T_{s}=N=2^{S F}$. This symbol, when sampled at Nyquist rate $\frac{T_{s}}{N}$, can be simply expressed as

$$
s^{(m)}[n]=\exp \left(j \pi n\left(\frac{n}{N}+\frac{2 m}{N}-1\right)\right),
$$

where $n \in\{0,1, . ., N-1\}$. In the following, we omit the superscript $(m)$ for clarity matter. Moreover, it must be noted that the preamble of the LoRa frame contains $K$ symbols (usually $K=8$ ) called raw chirps and corresponding to $m=0$, i.e. the instantaneous frequency of the raw chirp linearly increases from $-\frac{B_{w}}{2}$ to $\frac{B_{w}}{2}$. For any preamble, header, or data symbol, it is usual to assume the LoRa signal to be narrowband enough to model the channel as single scalar coefficient $h$ [11]-[14]. Under this assumption, and assuming a good time and frequency synchronization [8]-[10] (the effect of residual synchronization offset will be discussed in Section $\mathrm{V})$, the received symbol $r[n], n \in\{0,1, . ., N-1\}$, is obtained through

$$
r[n]=h s[n]+w[n],
$$

where $w[n]$ is the zero-mean complex additive white Gaussian noise (AWGN) with variance $\sigma^{2}$, which is supposed to be independent and identically distributed with respect to $n$. From (3), it is possible to estimate the transmitted $m$ value by considering the frequency bin corresponding to the maximum of the periodogram $|Y[k]|^{2}, k \in\{0,1, . ., N-1\}$, where $Y[k]=\operatorname{DFT}\left(r[n] \times s^{(0)}[n]^{*}\right)$, the superscript "(0)" indicating the raw chirp. Thus we have

$$
\hat{m}=\underset{k}{\arg \max }|Y[k]|^{2} .
$$

In practice, it is likely that the LoRa devices are located far from the gateway, or in deep underground, in such a way that the propagation channel cannot be modeled with a single coefficient anymore but as an actual multipath channel. Thus, we denote by $\mathbf{h}=\left[h_{0}, h_{1}, . ., h_{L-1}\right]$ the vector of the channel impulse response of size $L$. Furthermore, we define $i \in \mathbb{N}^{*}$ the index of the LoRa symbol within a frame. Then we rewrite (3) in matrix notation to consider the multipath channel $\mathbf{h}$ by means of the $N \times 1$ received signal vector $\mathbf{r}_{i}$ as

$$
\mathbf{r}_{i}=\underbrace{\left(\begin{array}{cccccc}
h_{L-1} & \cdots & h_{0} & 0 & \cdots & 0 \\
0 & \ddots & & \ddots & & 0 \\
\vdots & & \ddots & & \ddots & \vdots \\
0 & \cdots & 0 & h_{L-1} & \cdots & h_{0}
\end{array}\right)}_{\tilde{\mathbf{h}}} \tilde{\mathbf{s}}_{i}+\mathbf{w}_{i},
$$

where $\tilde{\mathbf{h}}$ is the $N \times(N+L-1)$ Toeplitz matrix containing the channel impulse response, $\tilde{\mathbf{s}}_{i}$ is the concatenation of the symbol $\mathbf{s}_{i}$ and the $L-1$ last samples of $\mathbf{s}_{i-1}$, which highlights the inter-symbol interference, i.e.

$$
\tilde{\mathbf{s}}_{i}=\left[s_{i-1}[N-L], . ., s_{i-1}[N-1], s_{i}[0], . ., s_{i}[N-1]\right]^{T} .
$$

Furthermore, $\mathbf{w}_{i}$ is the $N \times 1$ vector of complex additive white Gaussian noise with independent components $w_{i}[n] \sim$ $\mathcal{N}_{\mathbb{C}}\left(0, \sigma^{2}\right)$. It must be noted that (5) and (6) only hold if $i \geq 1$, highlighting why we omit the first symbol $i=0$. In the following, we take advantage of the cyclic properties of the symbols in the LoRa preamble to describe a frequency domain channel estimation process, similar to CP-OFDM methods. Moreover, both frequency domain equalization and demodulation are suggested. To this end, we first introduce Property 1 that gives the closed-form expression of the DFT of any modulated LoRa symbol when sampled at Nyquist rate.

Property 1. We define $S$ the vector corresponding to the DFT of $\boldsymbol{s}$ whose elements are given in (2). Thus, for any $m, k=$ $0,1, . ., N-1$, we denote by $S[k]$ the $k$-th element of $\boldsymbol{S}$, which can be expressed as

$$
\begin{aligned}
S[k]= & \sqrt{N} \exp \left(j \pi k\left(-\frac{k}{N}+\frac{2 m}{N}-1\right)\right) \\
& \times \underbrace{\exp \left(\frac{j \pi}{4}\right)(-1)^{m} \exp \left(-j \pi \frac{m^{2}}{N}\right)}_{\exp \left(j \theta_{m}\right)},
\end{aligned}
$$

where we defined $\theta_{m}=\frac{\pi}{4}+\pi m-\frac{\pi m^{2}}{N}$ for clarity purpose.

Proof. For any $m, n, k=0,1, . ., N-1$, the DFT of $s[n]$ is expressed as

$$
\begin{aligned}
S[k] & =\sum_{n=0}^{N-1} s[n] e^{-2 j \pi \frac{k n}{N}} \\
& =\sum_{n=0}^{N-1} \exp \left(j \pi \frac{n^{2}+n(2 m-N-2 k)}{N}\right) .
\end{aligned}
$$


We can identify the generalized quadratic Gauss sum [24] in (8). Since $N \neq 0$ and $2 k$ and $N$ are evenly valued, we can use Theorem 1.2.2 in [24] to obtain

$$
\begin{aligned}
S[k]= & \sqrt{N} \exp \left(\frac{j \pi}{4}\left(\operatorname{sgn}(N)-\frac{(2 m-2 k-N)^{2}}{N}\right)\right) \\
& \times \sum_{n=0}^{0} \exp \left(j \pi\left(-N n^{2}-n(2 m-N-2 k)\right)\right) \\
= & \sqrt{N} \exp \left(\frac{j \pi}{4 N}\left(N-(2 m-2 k-N)^{2}\right)\right) .
\end{aligned}
$$

After some straightforward developments, (9) leads to (7), which concludes the proof.

It is interesting to notice from (7) that the DFT of an upchirp is a downchirp with the additional phase offset $\theta_{m}$, and with constant modulus. To the best of our knowledge, this is an original result, that shows that frequency demodulation including channel estimation and equalization is possible in LoRa, such as detailed in Section III.

\section{FREQUency Demodulation IN LoRA}

This section is dedicated to the description of the demodulation of LoRa symbols in the frequency domain, allowing the receiver to cope with multipath channel featuring nonnegligible delays. We first present different preamble-based channel estimation methods, and then frequency domain equalization techniques.

\section{A. Frequency Channel Estimation}

We hereby consider LoRa symbols within the preamble, i.e. a succession of $K$ raw chirps (the sync word is not considered), where $K$ is usually set to $K=8$. Since the same symbols are used in the preamble, then for any $n \in$ $\{0, . ., N-1\}$ (and then especially for $n \in\{N-L, . ., N-1\}$ ) and $i \in\{1, . ., K-1\}$, we have $s_{i-1}[n]=s_{i}[n]$. As a consequence, similarly to the reception of the CP-OFDM signal after CP removal (see details in [25], chapter 1), we can rewrite (5) as

$$
\mathbf{r}_{i}=\underbrace{\left(\begin{array}{cccccc}
h_{0} & 0 & \cdots & h_{L-1} & \cdots & h_{1} \\
h_{1} & \ddots & \ddots & 0 & & h_{2} \\
\vdots & & \ddots & & \ddots & \vdots \\
\vdots & \ddots & & \ddots & & \vdots \\
0 & \cdots & h_{L-1} & \cdots & h_{0} & 0 \\
0 & \cdots & 0 & h_{L-1} & \cdots & h_{0}
\end{array}\right)}_{\overline{\mathbf{h}}} \mathbf{s}_{i}+\mathbf{w}_{i}
$$

where $\overline{\mathbf{h}}$ is the $N \times N$ circulant channel matrix, which is diagonal in the Fourier basis, defined by the fast Fourier transform (FFT) matrix F. We denote $\overline{\mathbf{H}}=\mathbf{F} \overline{\mathbf{h}} \mathbf{F}^{H}$ this diagonal matrix, where the diagonal is composed of the channel frequency response, whose $n$-th element $\bar{H}_{n, n}$ is defined as

$$
\bar{H}_{n, n}=\sum_{k=0}^{L-1} h_{k} e^{-2 j \pi \frac{k n}{N}} .
$$

Then we can rewrite (10) in the frequency domain as $\mathbf{R}_{i}=$ $\mathbf{F r}_{i}$, and using $\mathbf{F}^{H} \mathbf{F}=\mathbf{I}$ we obtain:

$$
\begin{aligned}
\mathbf{R}_{i} & =\mathbf{F} \overline{\mathbf{h}} \mathbf{F}^{H} \mathbf{F} \mathbf{s}_{i}+\mathbf{F} \mathbf{w}_{i} \\
& =\overline{\mathbf{H}} \mathbf{S}_{i}+\mathbf{W}_{i},
\end{aligned}
$$

where $\mathbf{S}_{i}=\mathbf{F} \mathbf{s}_{i}$ and $\mathbf{W}_{i}=\mathbf{F} \mathbf{w}_{i}$. It is noteworthy that (12) highlights that the received LoRa preamble symbols in frequency domain does not suffer from intersymbol interference (we do not consider interference from other signals). Equivalently to (12), we can rewrite (12) as

$$
\mathbf{R}_{i}=\overline{\mathbf{S}}_{i} \mathbf{H}+\mathbf{W}_{i},
$$

where $\mathbf{H}$ is the $N \times 1$ vector of the channel frequency response, and $\overline{\mathbf{S}}_{i}$ is the $N \times N$ diagonal matrix containing the frequency response of the chirp on its diagonal. Since the latter is known, the channel can be estimated through usual techniques used in multicarrier systems [18], [19]. In this paper, we present the least square (LS) and the minimum mean square error (MMSE) estimators [20], [21] adapted to LoRa signal as:

$$
\hat{\mathbf{H}}^{L S}=\overline{\mathbf{S}}_{i}^{-1} \mathbf{R}_{i}=\mathbf{H}+\overline{\mathbf{S}}_{i}^{-1} \mathbf{W}_{i},
$$

and

$$
\begin{aligned}
\hat{\mathbf{H}}^{M M S E}= & \mathbf{R}_{H}\left(\mathbf{R}_{H}+\sigma^{2}\left(\overline{\mathbf{S}}_{i} \overline{\mathbf{S}}_{i}^{H}\right)^{-1}\right)^{-1} \hat{\mathbf{H}}^{L S} \\
= & \mathbf{R}_{H}\left(\mathbf{R}_{H}+\frac{\sigma^{2}}{N} \mathbf{I}\right)^{-1} \hat{\mathbf{H}}^{L S},
\end{aligned}
$$

where $\mathbf{R}_{H}=\mathbb{E}\left\{\mathbf{H} \mathbf{H}^{H}\right\}$ is the channel covariance matrix, and I is the $N \times N$ identity matrix. It is worth emphasizing that $\mathbf{R}_{H}$ and $\sigma^{2}$ are a priori unknown at the receiver side. However, they can be substituted by fixed parameters or estimated thanks to methods described in [18]-[21].

To obtain (15), we used the property $\overline{\mathbf{S}}_{i} \overline{\mathbf{S}}_{i}^{H}=N \mathbf{I}$, which is directly drawn from Property 1 . It can be noted that $\hat{\mathbf{H}}^{L S}$ in (14) corresponds to the actual channel frequency response with additive noise distortion, while $\hat{\mathbf{H}}^{M M S E}$ is a "smoothed" version of $\hat{\mathbf{H}}^{L S}$ as it is filtered by $\mathbf{R}_{H}\left(\mathbf{R}_{H}+\sigma^{2} \mathbf{I}\right)^{-1}$, such as illustrated in Fig. 1. It shows the actual frequency response $|H|$ compared with $\left|\hat{\mathbf{H}}^{L S}\right|$ and $\left|\hat{\mathbf{H}}^{M M S E}\right|$. The parameters are SF7, the signal to noise ratio (SNR) is $\mathrm{SNR}=-10$ $\mathrm{dB}$, and the channel impulse response has been arbitrarily set to $\mathbf{h}=[1,0.1,1,0.1,1,1,1]$. It illustrates the sensitivity of the LS estimator to the noise, and the capability of the MMSE estimator to smooth $\hat{\mathbf{H}}^{L S}$ to recover $|\mathbf{H}|$, even in low SNR conditions. However, the main drawback of the MMSE channel estimator lies in its complexity. In fact, the matrix inversion and multiplication in (15) requires $\mathcal{O}\left(N^{3}\right)$ complex operations, even though it has been shown in [26], [27] that it is possible to reduce the complexity of the MMSE estimator to $\mathcal{O}(N \log (N))$. 


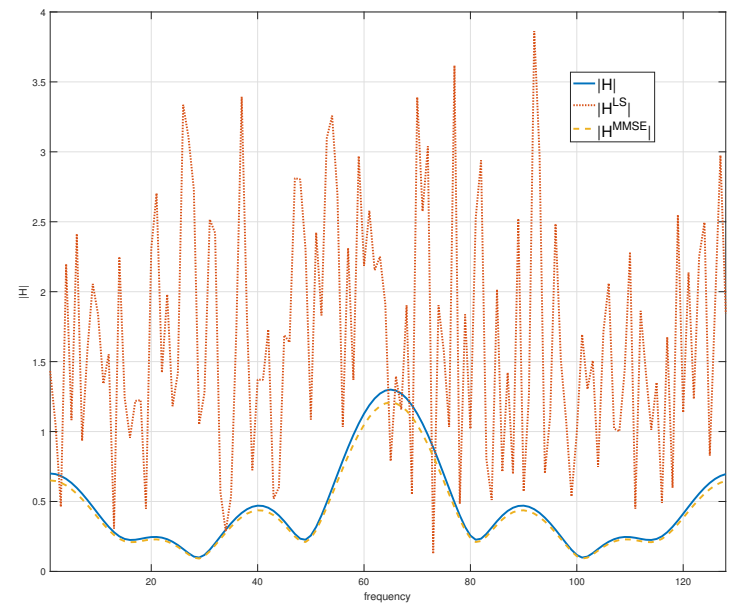

Fig. 1. Exact frequency response $|H|$ compared with the estimated ones $\hat{\mathbf{H}}^{L S}$ and $\hat{\mathbf{H}}^{M M S E}$.

To improve the LS channel estimator (with a lower complexity than MMSE), it is possible to take advantage of the $K$ symbols constituting the preamble in order to average the noise. Thus, even if the first symbol $i=0$ of the preamble does not match the cyclic property (therefore experiencing weak self-inteference), it can be considered in the average of the noise, leading to the new LS estimate (referred as K-LS) denoted by $\hat{\mathbf{H}}_{K}^{L S}$, obtained from (14) as:

$$
\begin{aligned}
\hat{\mathbf{H}}_{K}^{L S} & =\frac{1}{K} \sum_{i=0}^{K-1} \hat{\mathbf{H}}_{i}^{L S} \\
& =\frac{1}{K} \overline{\mathbf{S}}^{-1} \sum_{i=0}^{K-1} \mathbf{R}_{i},
\end{aligned}
$$

where $\overline{\mathbf{S}}$ contains the frequency response of a raw chirp. It must be noted that the second line in (16) has the same complexity as the usual LS estimator (14) in number of complex multiplications.

Interestingly, unlike CP-OFDM, the cyclic property of the symbols in LoRa, allowing to obtain (10), holds for any channel length $L$ as it does not depends on the CP duration. As a consequence, the performance of the channel estimation using LoRa preambles should not be influenced by the channel length $L$, as it is not limited by the size of the CP. Thus, the suggested channel estimation should cope with very long channel, which is relevant in LPWA applications. In fact, LPWA are constrained by large coverage cells (up to tens of kilometers radius) or deep underground transmissions that may lead to propagation multipath channels with long delays. This will be further illustrated and discussed in next sections.

\section{B. Frequency Domain Equalization}

In the following, we generally denote $\hat{\mathbf{H}}$ the vector of the channel estimate for any estimation method. We hereby suggest two frequency domain equalization techniques. Beforehand, it must be noted that the cyclic property inherent to the LoRa preamble symbols does not hold anymore in the general case of data symbols. Thus, (5) does not simplify to (10) and (13) as the terms of self and inter-symbol interference, denoted by $\mathbf{s}_{i}^{S I}$ and $\mathbf{s}_{i}^{I S I}$, respectively, must be considered. In a general case, (5) can be developed as

$$
\begin{aligned}
\mathbf{r}_{i} & =\tilde{\mathbf{h}} \tilde{\mathbf{s}}_{i}+\mathbf{w}_{i} \\
& =\overline{\mathbf{h}} \mathbf{s}_{i}+\mathbf{s}_{i}^{S I}+\mathbf{s}_{i}^{I S I}+\mathbf{w}_{i}
\end{aligned}
$$

where $\tilde{\mathbf{h}}$ and $\overline{\mathbf{h}}$ are the $N \times(N+L-1)$ and $N \times N$ channel matrices defined in (10) and (13), respectively, and

$$
\mathbf{s}_{i}^{S I}=-\left(\begin{array}{cccccc}
0 & 0 & \cdots & h_{L-1} & \cdots & h_{1} \\
0 & \ddots & \ddots & 0 & & h_{2} \\
\vdots & & \ddots & & \ddots & \vdots \\
\vdots & \ddots & & \ddots & & \vdots \\
0 & \cdots & 0 & \cdots & 0 & 0 \\
0 & \cdots & 0 & 0 & \cdots & 0
\end{array}\right) \mathbf{s}_{i}
$$

where

$$
\breve{\mathbf{s}}_{i}=\left[s_{i-1}[N-L], . ., s_{i-1}[N-1], 0, . ., 0\right]^{T} .
$$

It can be noticed that the matrix in (18) corresponds to the up right triangle of $\overline{\mathbf{h}}$, highlighting that the cyclic property does not hold anymore when considering data symbols. The frequency domain received data symbol can be expressed as

$$
\mathbf{R}_{i}=\overline{\mathbf{H}} \mathbf{S}_{i}+\mathbf{S}_{i}^{S I}+\mathbf{S}_{i}^{I S I}+\mathbf{W}_{i},
$$

where $\mathbf{S}_{i}^{S I}$ and $\mathbf{S}_{i}^{I S I}$ are the DFTs of $\mathbf{s}_{i}^{S I}$ and $\mathbf{s}_{i}^{I S I}$, respectively. Similarly to CP-OFDM where a simple one-tap per subcarrier is usually considered, we suggest, for a simplicity matter, to omit the interference terms and to apply a "one-tap per frequency bin" equalizer in order to remove the channel component $\overline{\mathbf{H}}$. Thus, for any $k=0,1, . ., N-1$ we define $Q[k]$ the zero-forcing equalizer coefficient, which we write

$$
Q[k]=\frac{\hat{H}[k]^{*}}{|\hat{H}[k]|^{2}+\varepsilon},
$$

where $\varepsilon$ is an additive constant avoiding the division by an extremely low value of $|\hat{H}[k]|^{2}$ if the latter is close to zero $(\varepsilon$ can be substituted by $\sigma^{2}$ to obtain the MMSE equalizer). The estimated symbol is then obtained with $\hat{\mathbf{S}}_{i}=\mathbf{Q} \mathbf{R}_{i}$ where $\mathbf{Q}$ is the $N \times N$ diagonal matrix containing the elements $Q[k]$. Alternatively to the equalizer in (22), we take advantage of the constant modulus of the LoRa symbol, i.e. $|S[k]|=1$, to suggest an equalization of the phase of $\mathbf{R}_{i}$ only. In that case, we denote by $\breve{\mathbf{S}}_{i}$ the equalized symbol, such that for any $k=0,1, . ., N-1$ its element $\breve{S}_{i}[k]$ is defined as

$$
\breve{S}_{i}[k]=\exp \left(j \arg \left(R_{i}[k]\right)\right) \exp (-j \arg (\hat{H}[k]))
$$




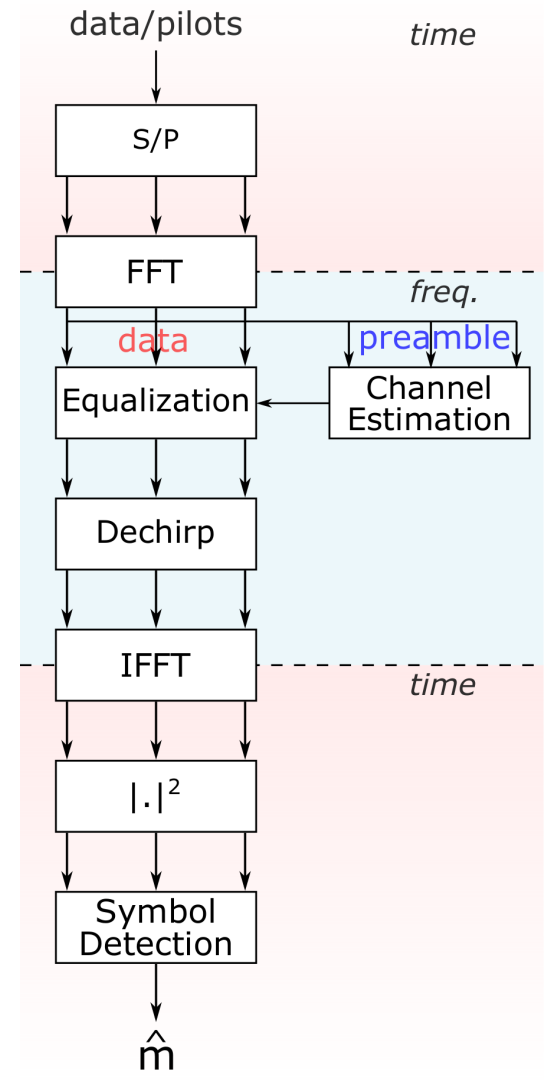

Fig. 2. LoRa Reception chain of the suggested frequency-domain channel estimation, equalization, and demodulation.

where $\arg ($.$) is the argument operator. The main advantage$ of the alternative equalization method in (23) is to avoid the computation of and the division by $|\hat{H}[k]|^{2}$ in (22). Moreover, we actually find that $\left|\breve{S}_{i}[k]\right|=1$ for any $k$ value. Once the received symbols have been equalized, the demodulation can be carried out directly in the time domain by using property 1 as follows:

$$
\hat{m}=\underset{n}{\arg \max }|\tilde{y}[n]|^{2} .
$$

where $\tilde{y}[n]=\operatorname{IDFT}\left(\hat{S}_{i}[k] \times S[k]^{(0)}\right)$, where $\hat{S}_{i}[k]$ can be substituted by $\breve{S}_{i}[k]$ if the alternative equalizer is considered, and the superscript $S[k]^{(0)}$ corresponds to (7) where $m=0$, i.e. the raw chirp in the frequency domain. In the following, we call argument equalizer (AE) the suggested method in (23).

Fig. 2 shows the LoRa reception chain that summarizes the suggested frequency-domain processes of channel estimation, equalization, and demodulation. The time and frequency domains are highlighted for a matter of clarity. Moreover, the "S/P" block indicates serial-to-parallel. Once again, we emphasize that such a reception chain is similar to those observed in multicarrier systems, in particular in OFDM. It must be noted that the complexity (in terms of complex multiplications) of the usual LoRa demodulation is mainly driven by the DFT, namely $\mathcal{O}(N \log (N))$ operations. Applying the proposed scheme only involves a slight increase of complexity to $\mathcal{O}(2 N \log (N))$. In the next section, we evaluate the suggested channel estimators and equalizers in LoRa system through simulations.

\section{Simulations Results}

The simulations results aim at evaluating the performance of the previously presented preamble-based LS and MMSE estimators in LoRa through the mean square error (MSE) of channel estimation defined as

$$
M S E=\frac{1}{N N_{r}} \sum_{i=0}^{N_{r}-1} \sum_{n=0}^{N-1}\left|\hat{\mathbf{H}}^{\text {est }}-\mathbf{H}\right|^{2},
$$

where "est" can correspond to LS, K-LS, or MMSE, and $N_{r}$ is the number of simulations runs. The performance of the equalization methods are presented using the bit error rate (BER) metric.

The simulations have been performed using matlab, and all the MSE results have been obtained by an average over $N_{r}=4000$ simulations runs, whereas the BER performance has been obtained through 80000 simulations runs where $m$ is uniformly chosen among $\{0,1, \ldots, N-1\}$. Unless specified otherwise, we consider LoRa signals with SF7 configuration, $B_{w}=250 \mathrm{kHz}$, and a preamble length of $K=8$. The channels have been modeled using a constant delay profile:

$$
\mathbf{h}=[\underbrace{\frac{1}{\sqrt{L}}, \frac{1}{\sqrt{L}}, . ., \frac{1}{\sqrt{L}}}_{L}],
$$

and we consider two channels:

1) channel 1 where $L=\frac{N}{16}$,

2) channel 2, where $L=\frac{N}{4}$.

It must be noticed that channel 2 is excessively long, as it corresponds to propagation a channel with a maximum delay of $1.28 \times 10^{-4} \mathrm{~s}$. Such a channel is not realistic, but it allows us to show the performance of the suggested frequency domain channel estimators in extreme propagation conditions.

Fig. 3 shows the MSE performance of the LS, K-LS, and MMSE estimators versus SNR in the range -15 to 0 $\mathrm{dB}$ for both channels 1 and 2 , and assuming that $\mathbf{R}_{H}$ is known. It can be observed that MMSE largely outperforms LS and K-LS thanks to the smoothing of the noise effect such as observed in Fig. 1. This result is consistent with that obtained in channel frequency estimation in multicarrier systems [18]-[21]. Focusing on the K-LS estimator, we notice that its MSE reaches a value that is eight times weaker than the LS. This is consistent with (16) as we deduce that the variance of the resulting noise in (16) is reduced by a factor $K$ (here $K=8$ ) compared with the noise affecting the LS estimate in (14). Moreover, such as expected, the same MSE performance is achieved for both channels, which highlights that the preamble-based frequency channel estimation in LoRa is not sensitive to the channel length.

Other series of simulations have been carried out to show the performance of both the channel estimation and the equalization methods. To this end, the BER performance is considered versus SNR (dB) in both Figs. 4 and 5. Fig. 4 shows the BER performance of LS, K-LS, and MMSE estimators, combined 


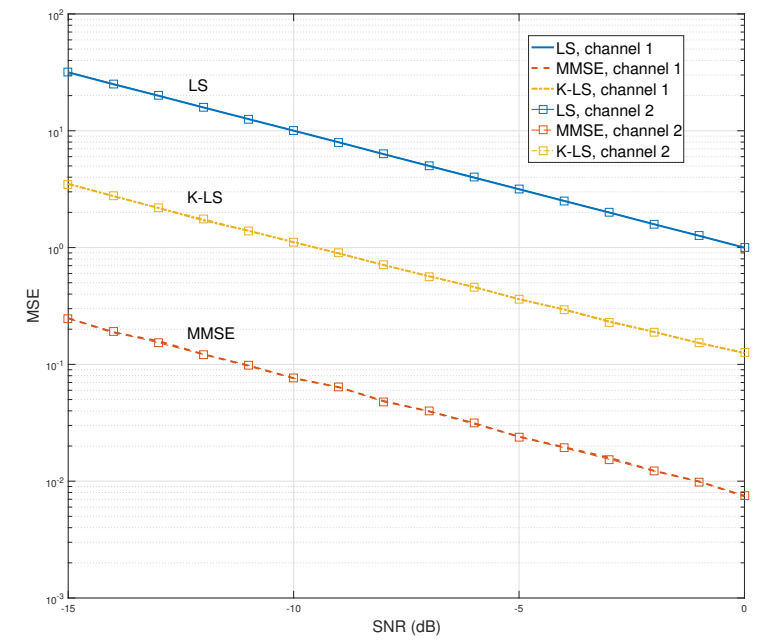

Fig. 3. MSE of channel estimation versus SNR (dB) for LS and MMSE channel estimators over channel 1 and channel 2, with known channel covariance matrix $\mathbf{R}_{H}$.

with $\mathrm{ZF}$ or AE equalizers considering the channel 1 (relatively short delays). The SNR range is -10 to $10 \mathrm{~dB}$. In addition, the case where the LoRa demodulation is performed without equalization is depicted as well, for reference purpose. The latter case leads to a constant BER of about 0.5 for any SNR value. This shows the need for an accurate channel estimation and equalization as soon as the channel cannot be considered as flat within the bandwidth $B_{w}$. According to the previously presented results in Fig. 3, it can be observed that, for a given equalizer and at a given BER level, the MMSE estimator outperforms the other ones by about $7 \mathrm{~dB}$ (LS) and $3 \mathrm{~dB}$ (K-LS). In addition, for any estimator, the performance of the AE is $1 \mathrm{~dB}$ weaker than that of the ZF. This reflects the non linearity of the $\mathrm{AE}$ in (23), which does not consider the received symbol module equalization unlike $\mathrm{ZF}$.

In Fig. 5, the same parameters as previously are considered, except that channel 2 is used, and the SNR range is from $-5 \mathrm{~dB}$ to $15 \mathrm{~dB}$. This shift toward larger SNR values compared with Fig. 4 is due to the higher severity of channel 2 compared with channel 1. Furthermore, it is worth noticing that the BER performance of the ZF equalizer reaches a lowest bound (e.g. $1.5 \times 10-3$ using MMSE estimator in the presented SNR range). This limit in the performance is mainly due to the effect of the interference terms $\mathbf{S}_{i}^{S I}$ and $\mathbf{S}_{i}^{I S I}$ in (21), when the latter are dominant against the noise term. On the contrary, the trajectories of the AE do not reach lowest bounds in the considered SNR range. Thus, we can see for the MMSE estimator that the AE outperforms the ZF equalizer for $S N R \geq 8 \mathrm{~dB}$. This shows the capacity of the AE to be more robust than $\mathrm{ZF}$ against the interference. This is an interesting property of the AE even if this holds in high SNR range.

\section{DISCUSSION}

It has been previously shown that it is possible to perform an efficient frequency domain channel estimation thanks to the

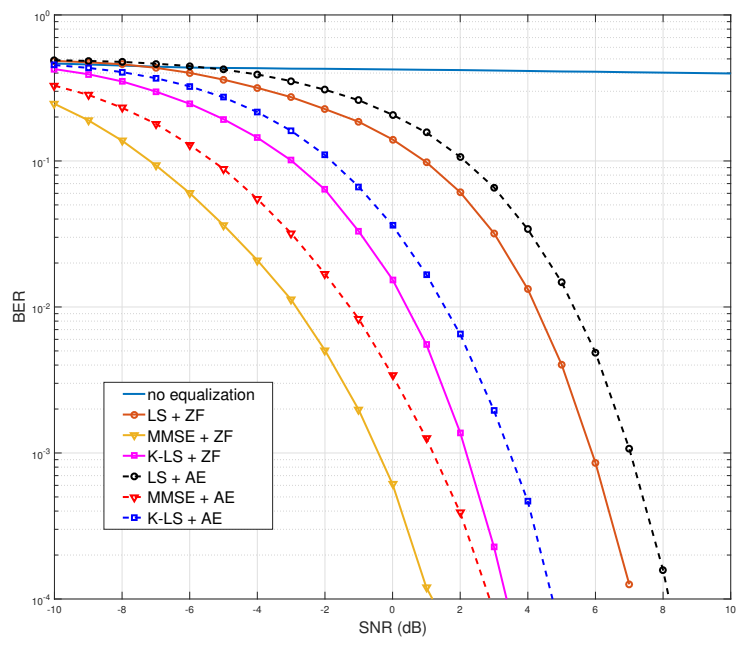

Fig. 4. BER versus SNR (dB) of both channel estimation and equalization techniques considering channel 1 .

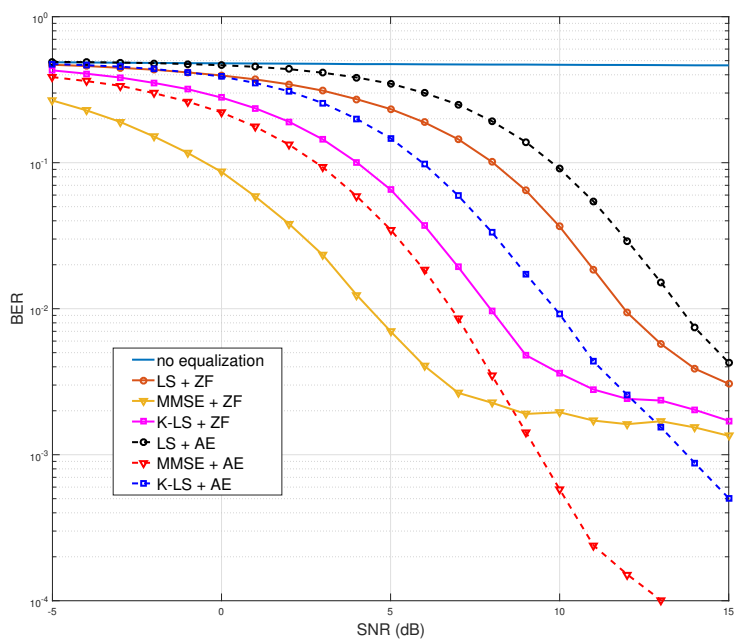

Fig. 5. BER versus SNR (dB) of both channel estimation and equalization techniques considering channel 2.

cyclic property of the symbols in the LoRa preamble. This section aims at discussing the obtained results and suggesting ideas and leads paving the way for further works.

The system model in Section II considers a perfectly synchronized reception which is an optimistic assumption since it is likely that synchronization errors remain, in particular at low SNR level. Thus, other series of simulations have been carried out to show the impact of the residual synchronization errors on the performance of the considered channel estimators. To this end, the signal $s_{i}$ in (2) is substituted by $s_{i}[n] e^{2 j \pi \delta_{f} T_{s} \frac{n}{N}}$, where $\delta_{f}$ is the residual frequency offset. In Fig. 6, we show the effect of $\delta_{f} \in\{0,15,30,60\} \mathrm{Hz}$ on the MSE performance of the LS, K-LS, and MMSE estimators. It can be observed that, in the considered SNR range, the offset has almost no impact on the performance of the LS estimation method, 


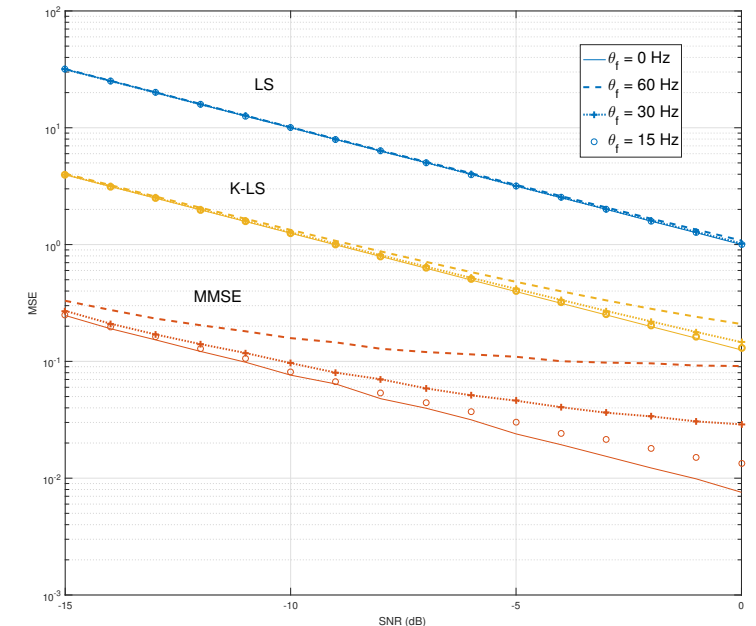

Fig. 6. MSE of channel estimation versus SNR (dB) for LS and MMSE channel estimators over channel 1. Effect of the frequency offset for $\theta=$ $\{0,15,30,60\} \mathrm{Hz}$.

because the AWGN is dominant in the deterioration of the performance. On the contrary, it can be observed that the performance of the K-LS and MMSE estimators are deteriorated as the offset increases, although they still outperform the LS estimator. Note that this could be improved by considering fractional offset synchronization methods such as described in [8]-[10].

It has been stated that a benefit of the preamble-based estimators presented in this paper relies in their performance which is independent of the channel length. However, unlike CP-OFDM modulation scheme, this feature holds only in the preamble, and other symbols (control and data symbols) of the LoRa frame are prone to self and intersymbol interference. The latter has been described in (21), and highlighted in Fig. 5 where the long channel 2 induces a BER performance lowest bound when the ZF equalizer is used. The suggested argument equalizer aims at reducing the effect of the interference and features a low complexity. Alternatively, it could be considered to remove (or at least reduce) the components $\mathbf{S}_{i}^{S I}$ and $\mathbf{S}_{i}^{I S I}$ by:

- Estimating (and removing) $\mathbf{S}_{i}^{I S I}$ thanks to the previously estimated LoRa symbol $\hat{\mathbf{s}}_{i-1}$ and the current channel. Note, however, that this may be impacted by the errors of estimation of $\hat{\mathbf{s}}_{i-1}$ and $\hat{\mathbf{H}}$.

- Processing a multi-tap equalizer instead of a single-tap one to reduce the self-interference $\mathbf{S}_{i}^{S I}$, such as proposed in OFDM system [28]. Note, however, that this kind of equalizer, in addition to the removal of $\mathbf{S}_{i}^{I S I}$, can be implemented to the cost of an increasing complexity, which may not be compatible with LoRa.

Furthermore, in case of mobility, the channel should be regularly tracked over the LoRa frame. This could be processed in a blind fashion through a stochastic gradient algorithm [29], or by reusing properly decoded symbols within the frame [17]. In the latter case, the performance of the channel estimation would be limited due to intersymbol interference.

\section{CONCLUSION}

In this paper, we propose frequency domain channel estimation and equalization methods for the LoRa IoT system. The estimation is based on the cyclic property of the received frame preamble sequence, which is similar to $\mathrm{CP}$ OFDM. From the expression of the received preamble chirp symbols sampled at the Nyquist rate, we take advantage of the cyclic nature of these symbols to derive an expression in the frequency domain that highlights the channel frequency response. Furthermore, we show that the received preamble symbols are not affected by intersymbol interference. We then propose the application of channel estimation techniques commonly used in CP-OFDM based systems. Moreover, two frequency domain equalizers are suggested. We provide simulation results showing the performance of the LS and MMSE channel estimators as well as for the equalizers, both in ideal frequency synchronization conditions and with a frequency offset between the transmitter and receiver ends. Finally, we discuss our results to suggest leads for future works.

\section{REFERENCES}

[1] G. A. Akpakwu, B. J. Silva, G. P. Hancke, and A. M. Abu-Mahfouz, "A Survey on 5G Networks for the Internet of Things: Communication Technologies and Challenges," IEEE Access, vol. 6, no. 12, pp. 3619 3647, December 2017.

[2] G. Pasolini, C. Buratti, L. Feltrin, F. Zabini, C. De Castro, R. Verdone, and O. Andrisano, "Smart city pilot projects using lora and ieee802.15.4 technologies," Sensors, vol. 18, no. 4, 2018. [Online]. Available: https://www.mdpi.com/1424-8220/18/4/1118

[3] M. Ji, J. Yoon, J. Choo, M. Jang, and A. Smith, "Lora-based visual monitoring scheme for agriculture iot," in proc. of 2019 IEEE Sensors Applications Symposium (SAS), Sophia Antipolis, France, March 2019, pp. 1-6.

[4] W. Ayoub, A. Samhat, F. Nouvel, M. Mroue, and J.-C. Prévotet, "Internet of Mobile Things: Overview of LoRaWAN,DASH7, and NB-IoT in LPWANs standards andSupported Mobility," in proc. of ICT'18, June 2018, pp. 1561 - 1581

[5] K. Mekki, E. Bajic, F. Chaxel, and F. Meyer, "A comparative study of LPWAN technologies for large-scale IoT deployment," ICT Express, vol. 5, no. 1, pp. 1 - 7, March 2019.

[6] "Lorawan ${ }^{\mathrm{TM}}$ specification," LoRa Alliance Technical Committee, October 2017, ver. 1.1 .

[7] L. Vangelista, "Frequency Shift Chirp Modulation: The LoRa Modulation," IEEE Signal Processing Letters, vol. 24, no. 12, pp. 1818 - 1821, December 2017.

[8] M. Xhonneux, D. Bol, and J. Louveaux, "A Low-complexity Synchronization Scheme for LoRa End Nodes," ArXiv, no. arXiv:1912.11344, pp. 1 - 10, December 2019.

[9] A. Marquet and N. Montavont, "Carrier and Symbol Synchronisation for LoRa Receivers,” in proc. of EWSN'20, Lyon, France, February 2020, pp. $1-6$.

[10] C. Bernier, F. Dehmas, and N. Deparis, "Low Complexity LoRa Frame Synchronization for Ultra-Low Power Software-Defined Radios," IEEE Transactions on Communications, vol. 68, no. 5, pp. 3140-3152, May 2020.

[11] T. Elshabrawy and J. Robert, "Closed-Form Approximation of LoRa Modulation BER Performance," IEEE Communications Letters, vol. 22, no. 9, pp. 1778 - 1781, September 2018.

[12] C. F. Dias, E. R. de Lima, and G. Fraidenraich, "Bit Error Rate ClosedForm Expressions for LoRa Systems under Nakagami and Rice Fading Channels," Sensors, vol. 19, no. 20, pp. 1 - 11, October 2019.

[13] V. Savaux and G. Ferré, "Simple Asymptotic BER Expressions for LoRa System over Rice and Rayleigh Channels," in proc. of WTS'21, San Francisco, CA, USA, April 2021, pp. $1-4$.

[14] V. Savaux, C. Delacourt, and P. Savelli, "Considering Sync Word and Header Error Rates for Performance Assessment in LoRa System," in proc. of WTS'21, San Francisco, CA, USA, April 2021, pp. 1 - 5. 
[15] H. R. Bapathu and S. S. Borkotoky, "The LoRa Modulation Over Rapidly-Varying Channels: Are the Higher Spreading Factors Necessarily More Robust?" in proc. of CCNC'21, Las Vegas, NV, USA, January 2021 , pp. $1-4$.

[16] Y. Guo and Z. Liu, "Time-delay-estimation-liked detection algorithm for lora signals over multipath channels," IEEE Wireless Communications Letters, vol. 9, no. 7, July 2020.

[17] S. Paquelet and P. Savelli, "Method for generating a pulse-positionmodulated signal, demodulation method, and corresponding computer program product and devices," WO Patent WO2 017211 553A1, 2017.

[18] M. K. Ozdemir and H. Arslan, "Channel Estimation for Wireless OFDM Systems," IEEE Communications Surveys and Tutorials, vol. 9, no. 2, pp. 18 - 48, 2nd Quarter 2007.

[19] Y. Liu, Z. Tan, H. Hu, L. J. Cimini, and G. Y. Li, "Channel Estimation for OFDM," IEEE Communication Surveys and Tutorials, vol. 16, no. 4, pp. $1891-1908$, Fourth quarter 2014.

[20] O. Edfords, M. Sandell, J.-J. van de Beek, S. K. Wilson, and P. O. Börjesson, "OFDM Channel Estimation by Singular Value Decomposition," IEEE Trans. on Communications, vol. 46, no. 7, pp. 931 - 939, July 1998.

[21] V. Savaux and Y. Louët, "LMMSE channel estimation in OFDM context a review," IET Signal Processing, vol. 11, no. 2, pp. 123 - 134, April 2017.

[22] X. Ouyang and J. Zhao, "Orthogonal chirp division multiplexing," IEEE Transactions on Communications, vol. 64, no. 9, pp. 3946-3957, 2016.

[23] M. Chiani and A. Elzanaty, "On the lora modulation for iot: Waveform properties and spectral analysis," IEEE Internet of Things Journal, vol. 6, no. 5, pp. 8463-8470, 2019.

[24] B. C. Berndt, R. J. Evans, and K. S. Williams, Gauss and Jacobi Sums. Ney York: John Wiley \& Sons, Inc., 1998, ch. 1: Gauss Sums, pp. $7-$ 56.

[25] V. Savaux, "Contribution to multipath channel estimation in OFDM context," Ph.D. dissertation, Supélec, November 2013.

[26] S. Ohno, S. Munesada, and E. Manasseh, "Low-complexity approximate LMMSE channel estimation for OFDM systems," in APSIPA ASC, Hollywood, CA, December 2012, pp. $1-4$.

[27] W. Zhou and W. H. Lam, "A Fast LMMSE Channel EstimationMethod for OFDM Systems," EURASIP Journal on Wireless Communications and Networking, March 2009.

[28] I. Barhumi, G. Leus, and M. Moonen, "Equalization for ofdm overdoubly selective channels," IEEE Transaction on signal processing, vol. 54, no. 4, April 2006.

[29] D. N. Godard, "Self-Recovering Equalization and Carrier Tracking in Two-Dimensional Data Communication Systems," IEEE Transactions on Communications, vol. com-28, no. 11, pp. 1867 - 1875, November 1980. 